\title{
One Lever Control for Variable Pitch Turboprop Aircraft
}

\author{
Giovanni Gaudio ${ }^{1}$, and Lorenzo Dambrosio ${ }^{1, *}$ \\ ${ }^{1}$ Department of Mechanics, Mathematics and Management, Politecnico di Bari, Via Orabona 4, 70125 \\ Bari, Italy
}

\begin{abstract}
The present work focuses on the control strategy concerning the propulsion system of a variable pitch turboprop aircraft. Extremely common solution for the management of the propulsion system of existing variable pitch turboprop aircraft considers a two-lever system for the control of both the turboprop output power and the propeller thrust. Such an approach translates, on one hand into a greater pilot responsibility and, on the other hand, into higher costs in terms of fuel consumption, aircraft maintenance and pilot training. In order to solve these drawbacks, an one-lever system for the control of the variable pitch turboprop aircraft propulsion system has been proposed. The design of this one-lever propulsion control has been carried at first by developing the whole aircraft ecosystem in Simulink (C) framework and then by implementing the control algorithm of the one-lever system.
\end{abstract}

\section{Introduction}

The aircraft flight dynamics is highly affected by the thrust generation mode produced by the propulsion system. From this point of view, the propulsion system has a great impact on the final aircraft mission. Generally speaking, the thrust generation relies on the momentum conservation principle [1]. This means that, in order to provide a given thrust, it is possible to vary the momentum of the propulsion system outlet gases performing either a significant speed variation on a limited gas flow rate or a modest speed variation on a large gas flow rate. The former approach is achieved by turbojet propulsion system which yields the best performance in terms of efficiency and thrust at quite high aircraft Velocity True Air Speed $(V T A S)$. These features are particularly appreciated when high aircraft VTAS is required. Fast military fighter planes employ these kinds of propulsion system [2].

On the other hand, the second approach is mainly achieved by propeller-based propulsion systems. Such aircrafts reach the best operating conditions at low VTAS. In particular, the variable pitch turboprop aircrafts are generally heavier and more complex with respect to turbojet ones with similar maximum engine power output. Nevertheless, since the turboprop aircrafts reach higher efficiency values in subsonic regime, they can produce quite important

\footnotetext{
* Corresponding author: ing.gaudio@gmail.com

*Corresponding author: lorenzo.dambrosio@poliba.it
} 
level of thrust at low speed, i. e., during the takeoff and climb phases. This makes such aircraft very attractive for cargo applications (high payload at average speed). This aspect translates in a number of advantages:

- $\quad$ since the air mass flow rate through the propeller is quite large and the required gases acceleration is modest, the thrust-specific fuel consumption of the turboprop aircrafts is smaller than that of turbojet ones;

- $\quad$ since the variable pitch turboprop aircrafts yield large thrust at low VTAS, they are able to take off and to land on shorter runways than is normally required turbojet-based aircrafts.

- $\quad$ since the propeller-based propulsion systems experience less severe conditions with respect to turbojet ones, the maintenance costs are lower [3].

Historically, the first turboprop aircrafts were equipped with fixed pitch propellers which were designed with a small angle of attack in order to allow a rapid takeoff; however, as a drawback, the maximum aircraft velocity during the cruise phase was limited. In order to increase the maximum aircraft velocity, keeping a high thrust value at low speed (rapid takeoff), these aircrafts have been equipped with variable pitch propellers [4]. As a consequence, the propulsion management system, called Constant-speed propeller, requires two independent control inputs (two-levers), to regulate at same time the gas turbine output power (power lever) and the propeller rotational speed (condition lever) [5]. Nevertheless, such an approach increases the pilot workload since he, not only, has to ensure the correct aircraft navigation, but, to keep the propeller maximum efficiency condition, he also has to provide the most appropriate values of the engine output power (power lever) and propeller rotational speed (condition lever). The degree of achievement of this latter task, which depends upon the pilot expertise, strongly affects the cruise fuel consumption. Furthermore, due to the complexity of the two-lever system the costs of the aircraft maintenance and of the pilot training are higher with respect to those of a fixed pitch propeller aircraft.

Due to all these considerations, a great interest has developed in recent years regarding the one-lever system for the management of the aircraft propulsion. Moreover, the one-lever approach results in quite evident advantages not only in terms of overall efficiency, but also for flight safety thanks to the reduction of maintenance operations on the engine.

\section{Variable pitch turboprop aircraft model}

The implementation of the proposed one-lever control system for the management of the aircraft propulsion system has been carried by developing the mathematical model of each aircraft component in the in Simulink (C) framework which allows an object-oriented approach. As a result, the Simulink (C) framework allowed to provide a whole aircraft ecosystem which simulates with an adequate level of accuracy the aircraft flight dynamics and the aerodynamic interactions of the aircraft with the external surroundings. Such a turboprop aircraft ecosystem mathematical model has been constituted by different modules that interact each other, as sketched in Fig. 1: the co-axial twin shaft gas turbine whose low pressure shaft drives the propeller; the aircraft trajectory module which acquires and develops the aircraft trajectory; the aircraft aerodynamic interactions module which takes into account the aircraft interactions with the external environment; an environment module which provides the environment conditions and allows to change the working conditions when the external conditions change. 


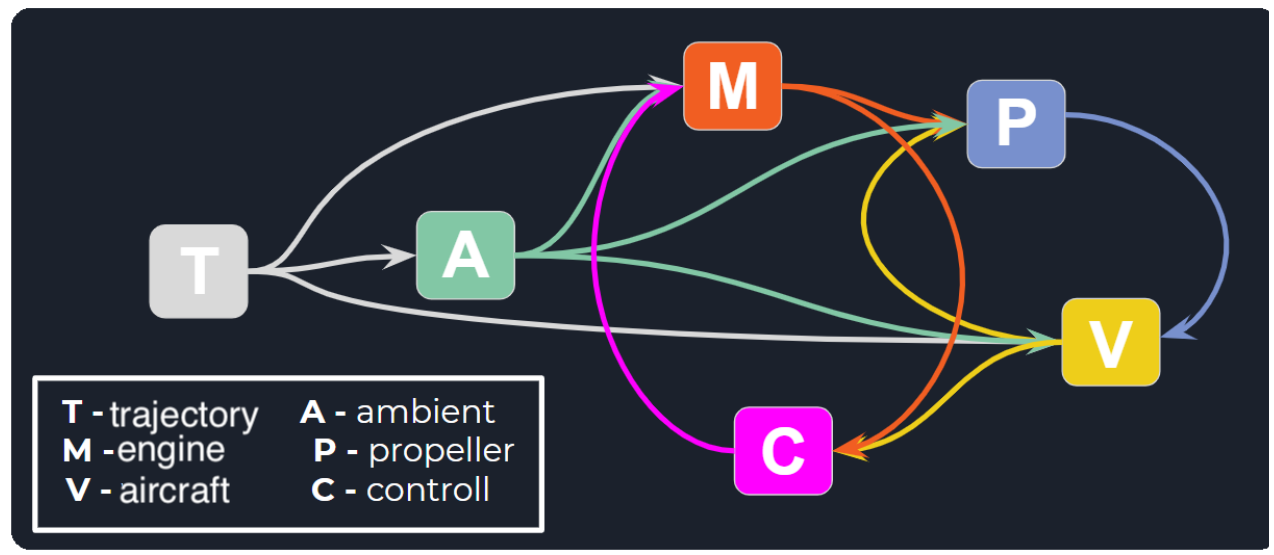

Fig. 1. Mathematical model logic scheme in Simulink@ framework.

Fig. 1 illustrates the logic interactions of each module and the model information flow of the aircraft system. For validation purpose, the aircraft system model outputs have been compared with a particular flight design condition (table 1), specified in [6].

Table 1. Flight design conditions.

\begin{tabular}{|c|c|}
\hline Design condition & Value \\
\hline Absolute Flight speed $[\mathrm{m} / \mathrm{s}]$ & 34.01 \\
\hline Altitude $[\mathrm{m}]$ & 0 \\
\hline Ambient air temperature $[\mathrm{K}]$ & 288.15 \\
\hline Ambient air pressure $[\mathrm{bar}]$ & 1.013 \\
\hline Compressor pressure ratio & 9.40 \\
\hline Air mass flow rate $[\mathrm{kg} / \mathrm{s}]$ & 4.18 \\
\hline Compressor thermal efficiency & 0.77 \\
\hline High pressure turbine speed $[\mathrm{rpm}]$ & 37500 \\
\hline
\end{tabular}

\subsection{Turboprop propulsion system}

From Ref. [7] the variable pitch turboprop propulsion system mathematical model has been implemented in Simulink (C) framework, including the propeller and gearbox model. Specifically, the gas turbine engine is a twin spool, where the low-pressure shaft drives the variable pitch propeller; it is composed by the inlet diffuser, the high-pressure turbine shaft, which drives the compressor, the combustion chamber, and the low-pressure power shaft, connected to the propeller. Each one of these components has been modeled according Ref. [8] and they have connected each other in the Simulink (C) environment, as shown in Fig. 2. In the same figure, it is possible to notice that the turboprop propulsion system block inputs are represented by the fuel mass flow rate, the low-pressure shaft load (propeller) and the ambient conditions; on the other hand, the block outputs are the mechanical power and the 


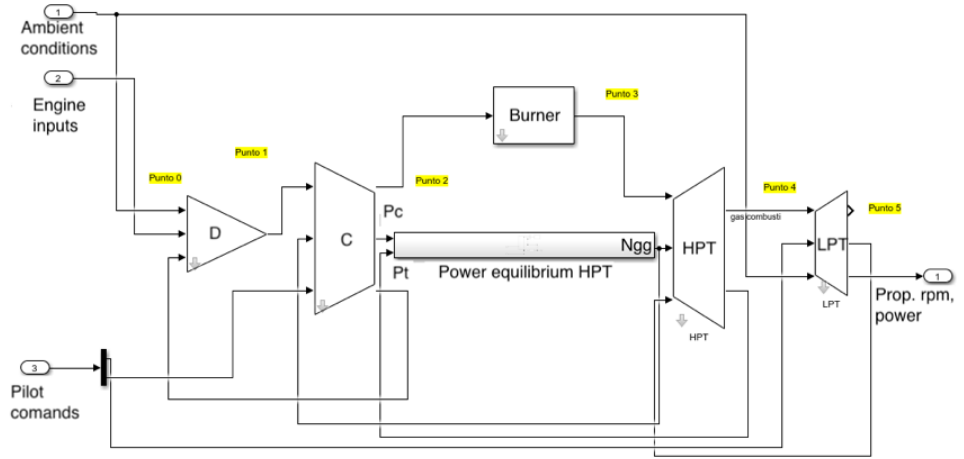

Fig. 2. Gas turbine engine scheme in Simulink $\odot$ framework.
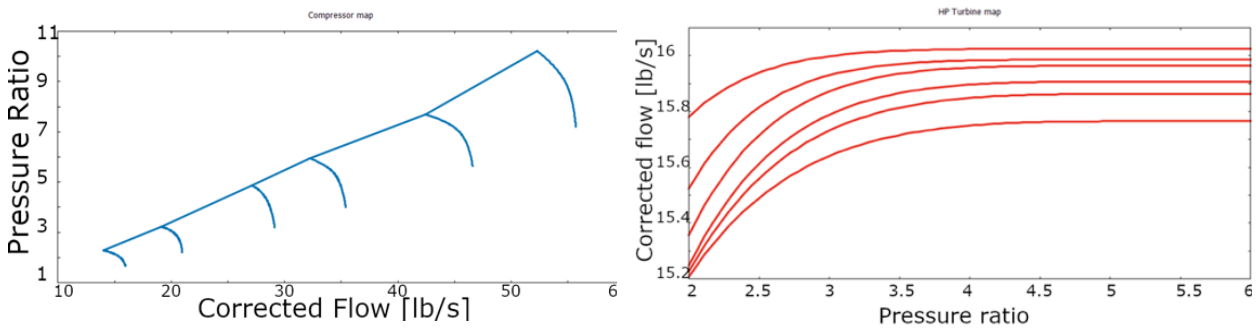

Fig. 3. Simulink@ compressor map.

Fig. 4. Simulink@ high pressure turbine map.
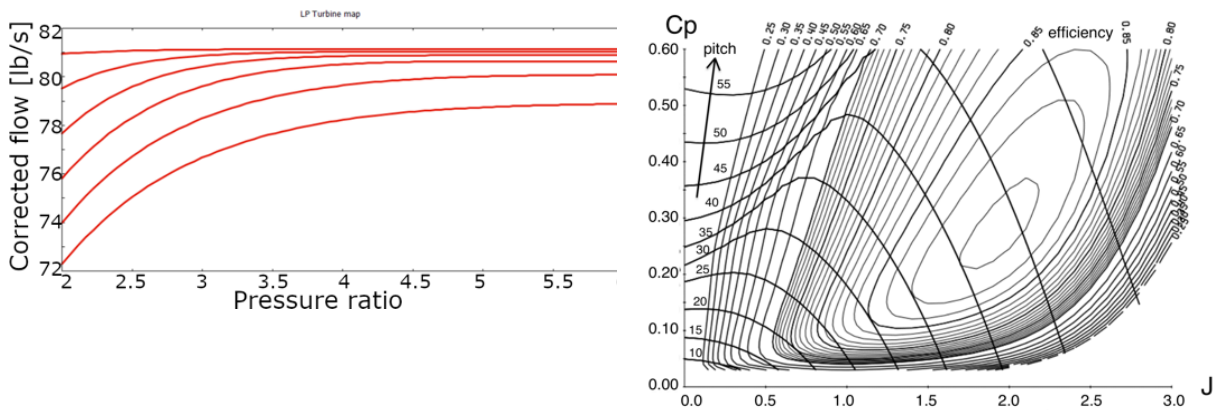

Fig. 5. Simulink@ low pressure turbine map.

Fig. 6. Simulink@ propeller map.

rotational speed of the low-pressure shaft. The diffuser block output variables are given by:

$$
T_{1}=M^{2} \cdot \frac{k_{a} R_{a i r} T_{0}}{2 c_{p m 01}} \quad p_{1}=p_{0} \cdot\left(\frac{T_{1}}{T_{0}}\right)^{\frac{k_{a}}{k_{a}-1}}
$$

where $M k_{a}$ and $R_{a i r}$ are the air Mach number, specific heat ratio and elastic constant, respectively, whereas $\mathrm{c}_{\mathrm{pm} 01}$ represents the mean constant pressure specific heat.

The compressor block employs the output of the diffuser block, which, in turn, receives the ambient conditions as inputs; moreover, the compressor needs data concerning the highpressure shaft in order to uniquely determine the working conditions of the compressor map. 
In this regard, since the actual maps of the turboprop system components (compressor, turbines and propeller) were no available, pre-generated generic maps, available in Simulink (C) libraries, have been employed. Figures 3- 6 provide the Simulink (C components (compressor, high pressure turbine, low pressure turbine and propeller) pre-generated map. As far as Fig. 6 is concerned $C_{P}$ and $J$ are the Power coefficient and the Advance ratio, respectively, defined as:

$$
C_{p}=\frac{P_{\text {prop }}}{\rho N^{3} D^{5}} \quad J=\frac{V}{N D}
$$

where $P_{P R O P}, N$ and $D$ represent the power, the rotational speed and tip diameter of the propeller; on the other hand, $\varrho$ and $V$ are the air density and the aircraft velocity. Of course, in order to tailor such generic maps to each component, a map scaling process has been carried out [9]. From the compressor map, the output variables are given by:

$$
\beta_{c}=\frac{p_{2}}{p_{1}} \quad T_{2}=T_{1}\left[1+\frac{\beta_{c}^{\frac{R_{a i r}}{c_{p m 12}}}-1}{\eta_{i s, c}}\right] \quad P_{c}=\frac{\dot{m}_{2} L_{c}}{\eta_{m t}}
$$

being $\beta_{c}, T_{2}$ and $p_{2}$ the compression ratio, and the outlet air temperature and pressure, respectively. On the other hand, $\eta_{i s, c}, \dot{m}_{2}$ and $P_{c}$ indicate the compressor isentropic efficiency, the mass flow rate and the requested power. Considering the burner block, the outlet gas temperature, pressure and mass flow rate, $T_{3}, p_{3}$ and $\dot{m}_{3}$ can be determined as:

$$
f=\frac{\dot{m}_{b}}{m_{a i r}}=\frac{h_{g c}\left(T_{3}\right)-h_{a i r}\left(T_{2}\right)}{h_{b}\left(T_{2}\right)+\eta_{b} H_{i}-h_{g c}\left(T_{3}\right)} \quad p_{3}=p_{2} \cdot y_{b} \quad \dot{m}_{3}=\dot{m}_{2}+\dot{m}_{b}
$$

where $f$ is the air-fuel ratio, $m_{a l r}$ and $\dot{m}_{b}$ indicate the air and the fuel mass flow rates whereas $h_{g c}, h_{a i r}$ and $h_{b}$ represent the gas, air and fuel specific enthalpy, respectively. Moreover, $\eta_{b}$ is the burner efficiency, $H_{i}$ is the fuel Lower Heating Value and $\mathrm{y}_{\mathrm{b}}$ represents the combustor pressure loss. From the burner block outlet variables, it is possible to determine the gas conditions at the high-pressure turbine inlet (Fig. 7), which, in turn, (after tuning the highpressure turbine map) permits to determine the high-pressure turbine output power by using:

$$
\beta_{H P T}=\frac{p_{4}}{p_{3}} \quad T_{4}=T_{3}\left\{1+\eta_{t}\left[\left(\beta_{H P T}\right)^{\frac{R g c}{c_{p g c\left(T_{m 34}\right)}}}\right]-1\right\} \quad P_{H P T}=\dot{m}_{4} \cdot L_{H P T} \cdot \eta_{m t}
$$

being $\beta_{H P T}, T_{4}$ and $p_{4}$ the high-pressure turbine expansion ratio, and the outlet gas temperature and pressure, respectively. Moreover, $\eta_{t}$ and $\eta_{m}$ indicate the high-pressure turbine isentropic and mechanical efficiency, respectively, whereas $R_{g c}$ and $\dot{m}_{4}$ represent the gas elastic constant and mass flow rate. At this point, considering the high-pressure shaft dynamical equilibrium, the compressor and high-pressure turbine blocks are able to evaluate their respective working point variations.

As far the low-pressure turbine is concerned, the working conditions can be determined considering the equilibrium of the low-pressure shaft which depends upon, on one hand, the output variables coming from the high-pressure turbine block, i.e. gas temperature, pressure and mass flow rate, and, on the other hand, the torque demanded by the propeller. The lowpressure turbine main equations are given by:

$$
T_{5}=T_{4}-\frac{c_{p m 45^{\prime}}}{c_{p m 45}}\left(T_{4}-T_{5^{\prime}}\right) \eta_{t, E L} \quad p 5=p_{4}\left(\frac{T_{5^{\prime}}}{T_{4}}\right)^{\frac{c_{p m 45^{\prime}}}{R_{g c}}} \quad P_{a x, t}=\dot{m}_{3}(1+f) \cdot c_{p m 45}\left(T_{4}-T_{5}\right) \eta_{m t, E L}
$$




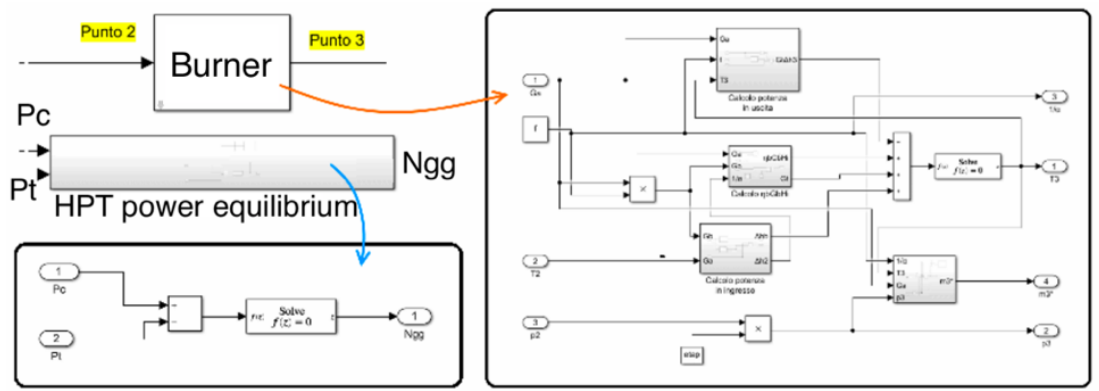

Fig. 7. Burner and high-pressure turbine in Simulink@ framework.

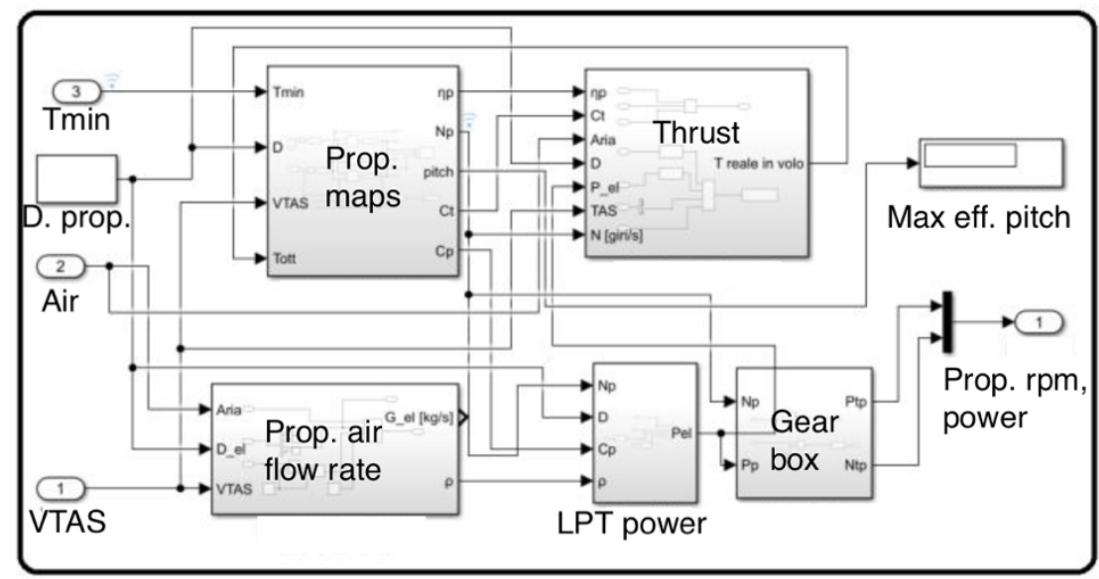

Fig. 8. Propeller and gearbox scheme in Simulink® framework.

being $T_{5}$ and $p_{5}$ the low-pressure turbine inlet gas temperature and pressure, respectively, whereas, $\eta_{t}$ and $\eta_{m t, E L}$ indicate the low-pressure turbine isentropic and mechanical efficiency. Finally $\mathrm{P}_{\mathrm{ax}, \mathrm{t}}$ represents the output low-pressure turbine power.

In order to achieve the target rotational speed, the torque produced by the low-pressure turbine has to equal to that opposed by the external load which is represented by the propeller (see Eqs. (2)). Specifically, the propeller, which is connected to the low-pressure shaft by means of a gearbox, is responsible for the production of almost the entire thrust needed for the aircraft to fly (a residual portion of the thrust is supplied by the gas ejection nozzle located in the rear section of the gas turbine engine). In order to evaluate the low-pressure shaft working conditions, it necessary determine the thrust required to maintain the aircraft to a given VTAS. In fact, for given VTAS, ambient conditions, airfoil angle of attack and propeller rotational regime, the propeller thrust and drag (propeller resistance torque) are uniquely defined. As illustrate in Fig. 8, such an information is provided by a specific block embedded in the propeller block that, considering the external air condition (yielded by the block Ambient), carries out the aircraft translation equilibrium. Since the demanded thrust is directly affected by the propeller blades angle of attack, which, in turn, depends by the blades pitch angle, it is possible to state that blades pitch angle determines propeller working 
condition or, in other words, it defines the equilibrium value of the propeller rotational speed (proportional to the low-pressure shaft rotational speed). Nevertheless, there is not a unique value for the pitch angle that provides the necessary thrust level for a specific flight condition. The reason is because the pitch angle can be varied within an interval providing the same thrust level, as long as the propeller rotational speed is accordingly modified. Of course, different blades pitch angle - propeller rotational speed configurations do not have the same performance in terms of airfoil efficiency, propeller promptness response and control effort. In this perspective, during the flight operations, the most experienced pilots, using two-levers commands, can use different approaches, trying to optimize one of the mentioned criteria; on the contrary, the less experienced ones just carry out the flight operations with no particular attention to the employed approach. In the present paper the blades pitch angle - propeller rotational speed configuration has been chosen in order to make sure that during the aircraft operations the propeller blades maintain the maximum airfoil efficiency. Such a configuration has been selected considering the turboprop propulsion system mathematical model as the model reference of a model-based controller [6][7]. This choice represents a first step solution in order to evaluate the feasibility of the one-lever approach with respect the traditional two-lever one.

For the aforementioned reasons, the input variables of the propeller block are given by the aircraft VTAS and the ambient conditions whereas the output ones are represented by the propeller needed power and the low-pressure shaft rotational speed.

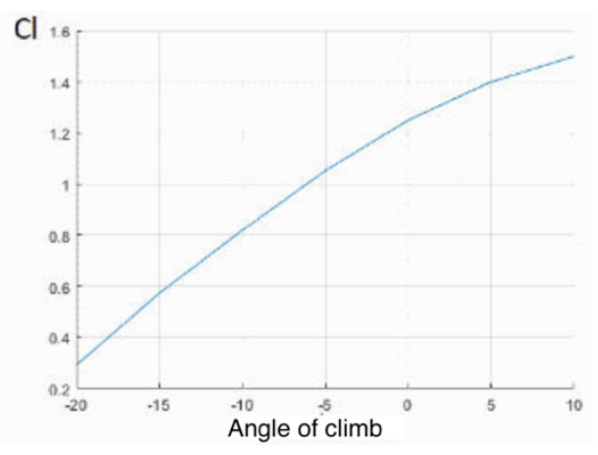

Fig. 9. Lift $S A A B 340$ characteristics.

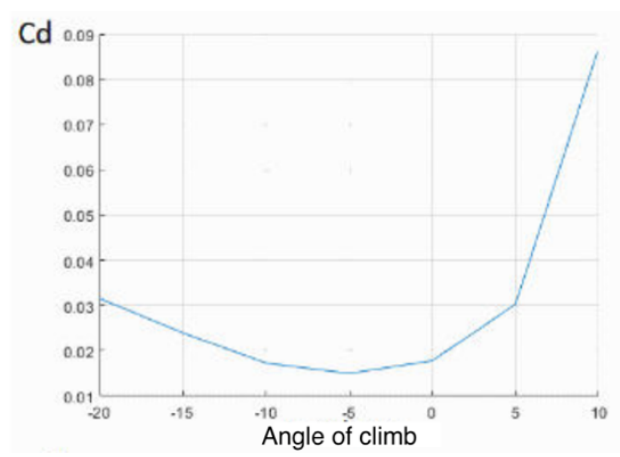

Fig. 10. Drag SAAB 340 characteristics.

\subsection{Aircraft dynamics}

As stated in the previous section, in order to keep the aircraft in stationary flight, it has been necessary to determine the adequate level of thrust. This assignment has been carried out by the aircraft dynamic block which considers the aircraft equilibrium in a vertical plane flight. Therefore, it has been necessary to provide specific information concerning the aircraft; in this paper, the SAAB 340 aircraft has been chosen as study case, not only because this aircraft features and mission well matches the modelled turboprop propulsive system but also for the data availability in terms of aircraft characteristics and flight data. The equilibrium equations in vertical plane flight not only involve the propulsive system thrust and the aircraft weight, but also the entire aircraft lift and drag are necessary. Since the present work focuses on the control of the turboprop propulsive system and an accurate calculation model for the aircraft lift and drag would be beyond the scope of this paper, only educated estimation [10] for the aircraft lift and drag have been provided. In this perspective, Figures 9 and 10 illustrates realistic lift and drag characteristics for the SAAB 340 aircraft. In order to complete the whole aircraft ecosystem, it is convenient to provide the ambient conditions for each aircraft cruise 
velocity and altitude. This assignment is accomplished by the Ambient block that implements, from Ref. [11], the mathematical model for the calculation of the air thermodynamic conditions.
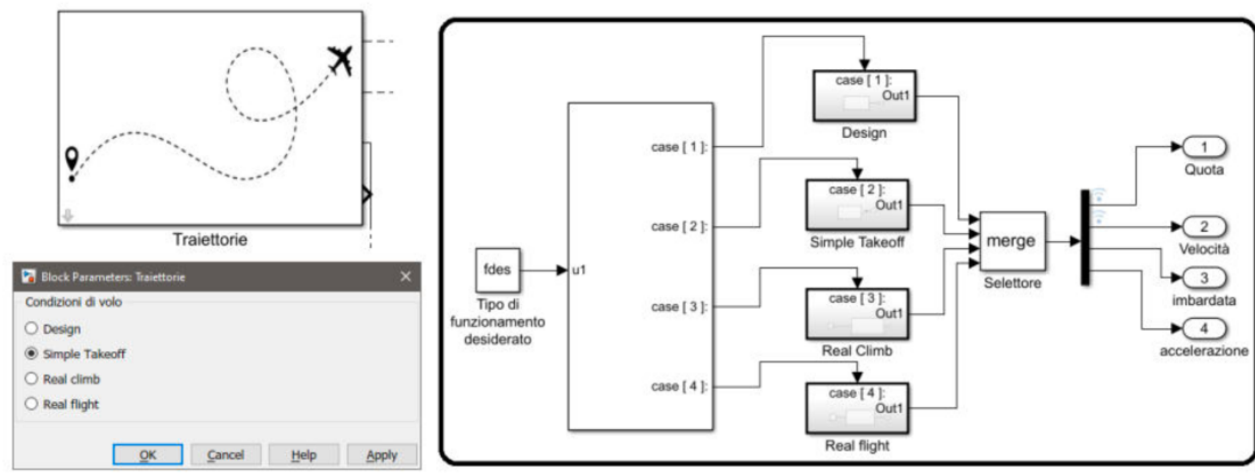

Fig. 11. Trajectory block scheme in Simulink $₫$ framework.

\section{Results}

In this section will be presented the performance results regarding the application of the proposed one-lever approach for the control of the turboprop propulsion system. In particular, two different flight maneuvers have been considered and both of them have been carried out by using the proposed one-lever approach. For comparison purposes it would be interesting to execute such maneuvers adopting the traditional two-lever approach; unfortunately, these data are not easy to find. Nevertheless, some concluding remarks can still be made.

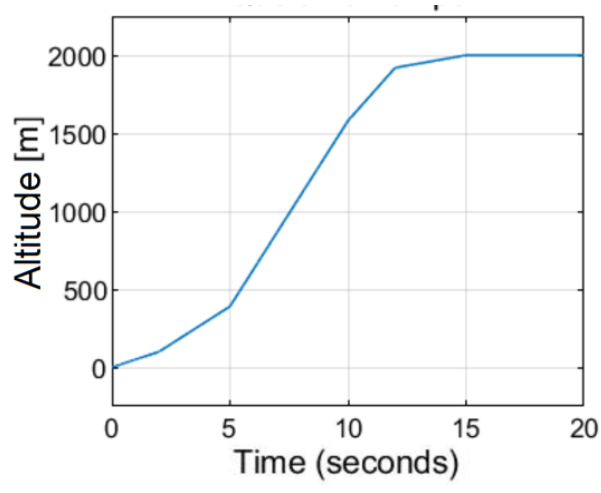

Fig. 12. Simple Takeoff - aircraft altitude vs. Time.

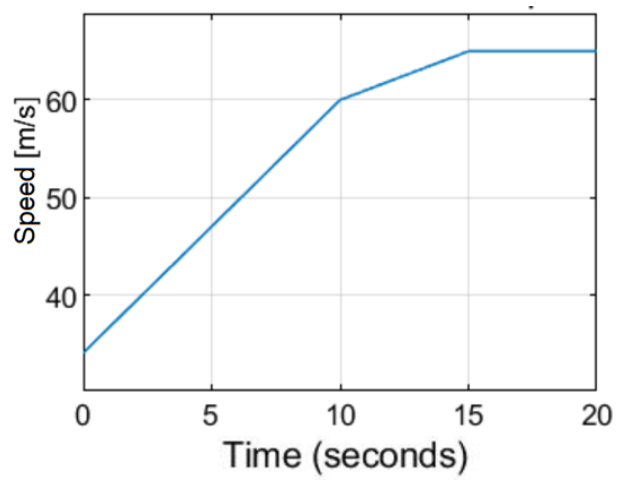

Fig. 13. Simple Takeoff - aircraft velocity vs. Time.

As mentioned earlier, in order to perform such maneuvers an aircraft reference as study case has been selected: the SAAB 340. The reasons behind this choice not only are due to the features and mission of this aircraft that well matches the modeled turboprop propulsive system but also lies on the data availability in terms of aircraft characteristics and flight data. This latter aspect allowed to build the block Trajectory which, in turn, has been able to provide the realistic trajectory data in terms of altitude, aircraft speed, angle of climb and aircraft acceleration for the SAAB 340 main maneuvers in vertical plane flight (Fig. 11). 


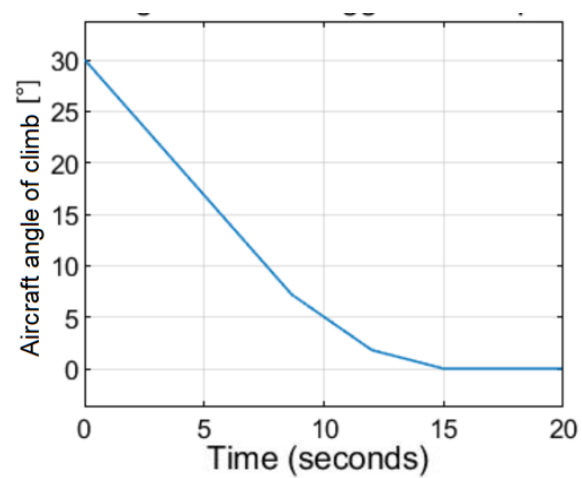

Fig. 14. Simple Takeoff - aircraft angle of climb vs. Time.

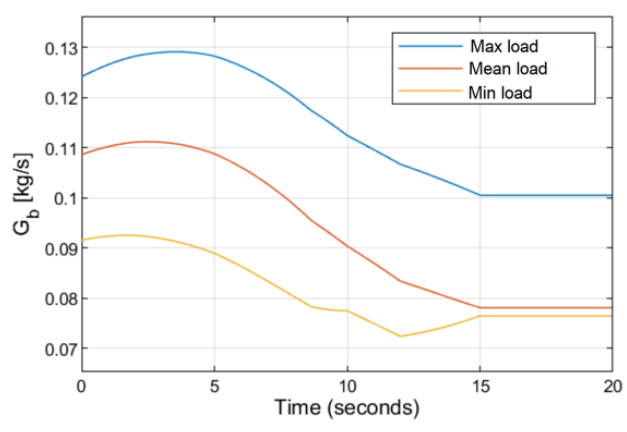

Fig. 15. Simple Takeoff - aircraft fuel consumption vs. Time.

The first considered flight maneuver is the Simple Takeoff which neglects the runway acceleration and consider the aircraft with an initial velocity equal to $34.04 \mathrm{~m} / \mathrm{s}$ reaching a final level flight condition at the altitude of $2000 \mathrm{~m}$, as shown in Figures 12, 13 and 14. This maneuver has been carried out considering three different payload conditions: minimum load $(8036 \mathrm{~kg})$, medium load $(10483 \mathrm{~kg})$ and maximum load $(12930 \mathrm{~kg})$. Fig. 15 summarizes the required fuel mass flow rate for the three different payload conditions. It is noteworthy that for the two-lever approach, during the Simple Takeoff maneuver, the power lever is set in takeoff position which corresponds to the maximum power request from the gas turbine engine. Consequently, the required fuel mass flow rate still stands at maximum level regardless of the considered payload. From Fig. 15, the single lever solution not only results in a fuel saving at maximum payload condition with respect the two-lever approach, but it also permits to adapt the fuel consumption to the actual payload condition. Figures 16, and 17 illustrate the propeller thrust and pitch angle. From the former figure, it is possible to notice that the one-lever solution considers that the necessary thrust not only reduces when the payload decreases, but, for a given payload, it experiences a lowering trend; on the other hand, considering the two lever approach, since the power lever is set in takeoff position (maximum low pressure turbine power), it easy to conclude that the thrust level time behavior is fairly higher than the one corresponding to the one lever approach. This aspect confirms that the one lever method offers a more efficient control of the turbo- prop propulsive system with respect the two-lever one. As far the blade propeller pitch angle is concerned, Fig. 17 shows the time history variation related to the three payloads condition. The blade pitch angle represents the manipulating (control) variable which ensure the maximum efficiency condition for the blade airfoil. Fig. 18 reports the gas turbine high pressure and low-pressure rotational speed corresponding, for conciseness, just for the maximum load conditions. Focusing on the low-pressure rotational speed, it is remarkable that these time trends are coupled to those correlating to the blade pitch angle (at respective load condition, Fig. 17) in order to maintain the maximum airfoil efficiency during the maneuver. For completeness, Fig. 19 reports, for the one-lever approach, the two-lever equivalent position during the Simple Takeoff maneuver with maximum payload.

The second maneuver is the Simple Descent and, similarly to the previous test case, it takes place in a vertical plane. Figures 20, 21 and 22 illustrate the main aircraft trajectory flight parameters of the Simple Descent maneuver, i. e., aircraft altitude, velocity and angle of climb. Again, the minimum, medium and maximum payload conditions have been tested. Likewise, to the Simple Takeoff case, Fig. 23 depicts the fuel mass flow rate for the Simple Descent maneuver. Nevertheless, unlike the Simple Takeoff, since in the present case the 
pilot maneuver data are not available, it is not possible to compare the one lever results with those deriving from the two-lever approach.

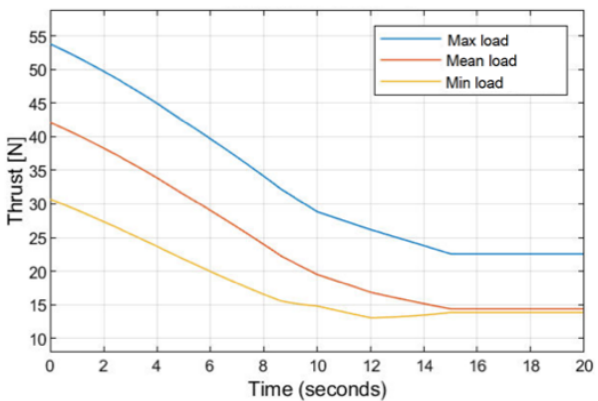

Fig. 16. Simple Takeoff - aircraft propeller thrust vs. Time.

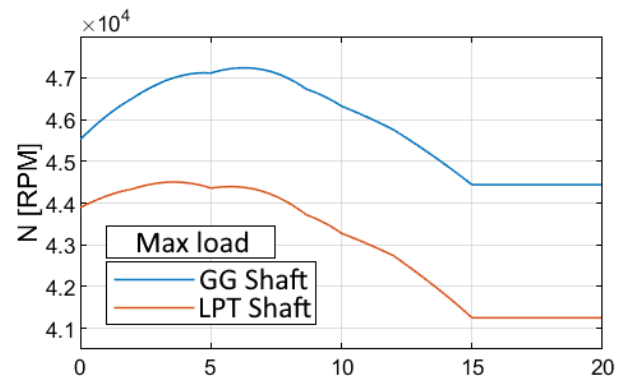

Fig. 18. Simple Takeoff - Gas turbine shaft speed vs. Time.

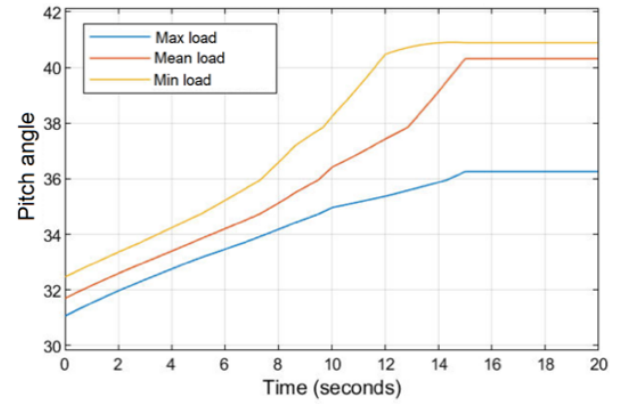

Fig. 17. Simple Takeoff - aircraft propeller pitch angle vs. Time.

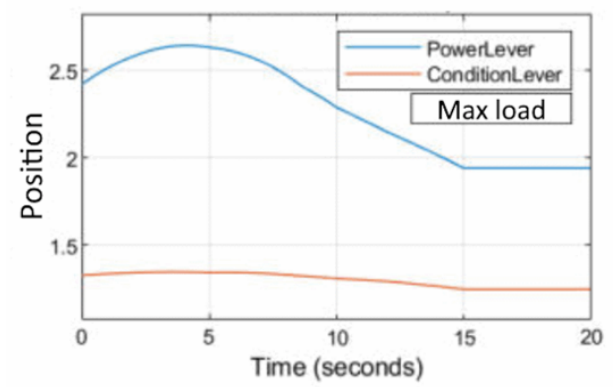

Fig. 19. Simple Takeoff - two-lever equivalent position vs. Time.

However, considering the two-lever approach, different pilots would achieve the same flight operation (Simple Descent) with different performance in terms of airfoil efficiency, propeller promptness response and control effort. From a cursory look at Fig. 23, also in this case, the one lever method adjusts the fuel consumption to the actual payload condition. Accordingly, figures 24 , and 25 show the propeller thrust and pitch angle at each payload condition. Again, these time trends reflect the particular maneuver imposed by the pilot and, for each payload condition, a distinct trust and pitch angle time behavior is indicated. This latter consideration allows to remark that the proposed one-lever controller for the turboprop propulsion system is not an autopilot. On the contrary, it supports the pilot during the maneuver providing the requested power in maximum propeller efficiency condition. In fact, if the pilot had performed a different Simple Descent (Glide) maneuver, according figures 20, 26 and 27, the one-lever controller would respond with different values for fuel mass flow rate, thrust and blade propeller pitch angle with respect to those illustrated in figures 23, 24 and 25. For conciseness, concerning the Glide Simple Descent maneuver, only fuel mass flow rate time history has been reported in Fig. 28. This latter result confirms that the proposed one lever controller is able to manage the propulsive system in order permitting the pilot to focus on the flight maneuver. 


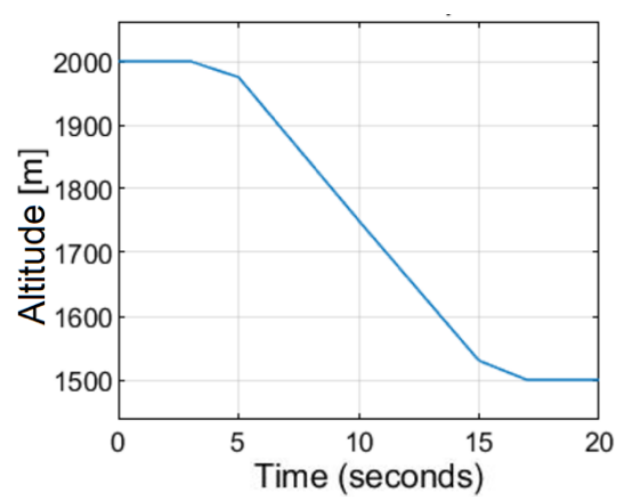

Fig. 20. Simple Descent - aircraft altitude vs. Time.

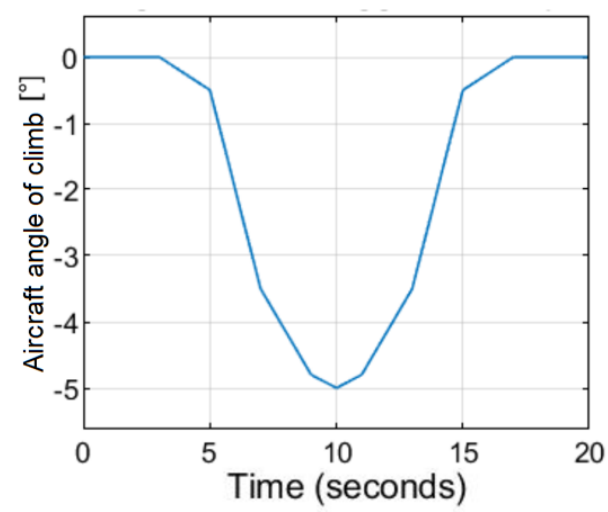

Fig. 22. Simple Descent - aircraft angle of climb vs. Time.

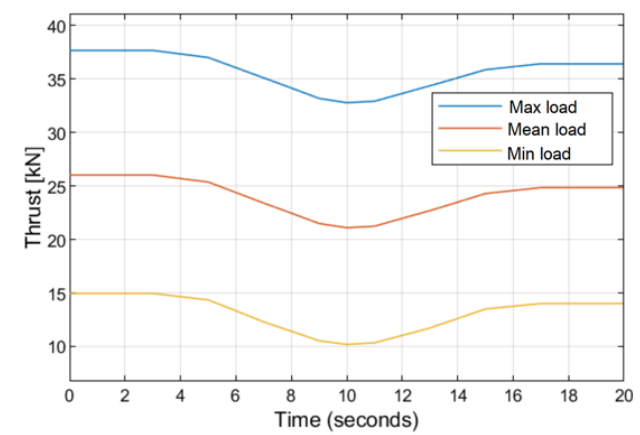

Fig. 24. Simple Descent - aircraft propeller thrust vs. Time.

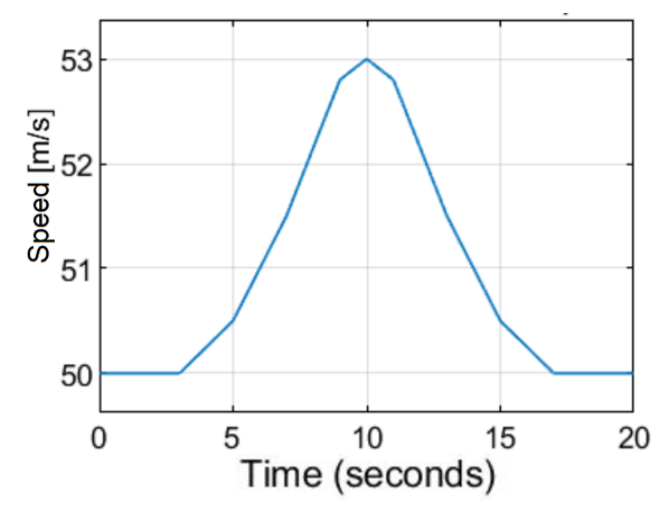

Fig. 21. Simple Descent - aircraft velocity vs. Time.

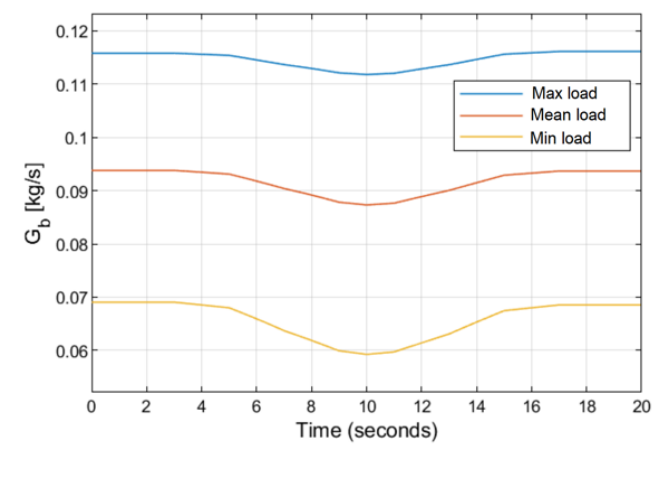

Fig. 23. Simple Descent - aircraft fuel consumption vs. Time.

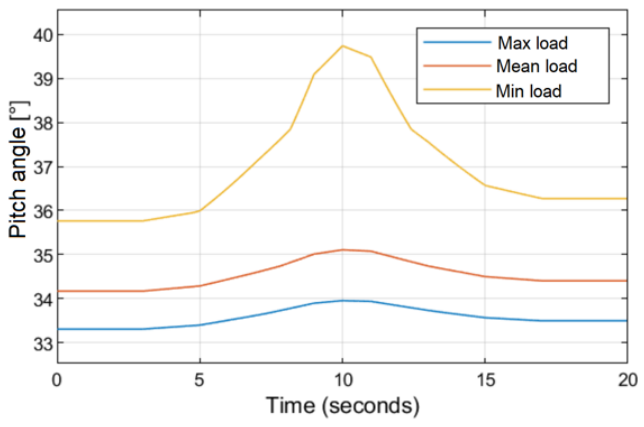

Fig. 25. Simple Descent - aircraft propeller pitch angle vs. Time.

\section{Conclusions}

In the present work the control strategy concerning the propulsion system of a variable pitch turboprop aircraft has been considered. Most of the variable pitch turboprop aircrafts adopt for the managing of the propulsion system the well settled solution composed by a two-lever system for the control of both the engine output power and the propeller thrust. The twolevers system requires, on one hand, a greater pilot expertise since he, not only, has 


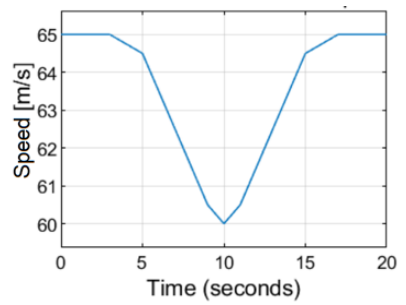

Fig. 26. Glide Simple Descent - aircraft velocity vs. Time

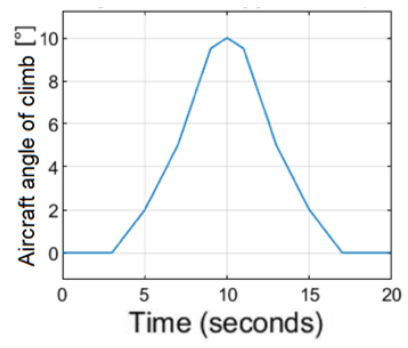

Fig. 27. Glide Simple Descent - aircraft angle of climb vs. Time

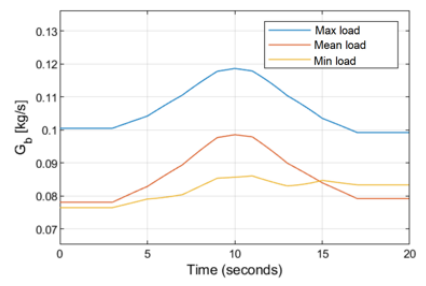

Fig. 28. Glide Simple Descent aircraft fuel consumption vs. Time

to ensure the correct aircraft navigation, but, in order to keep the propeller maximum efficiency condition, he also has to provide the most appropriate values of the engine output power (power lever) and of the propeller rotational speed (condition lever); on the other hand, the two-lever control management results in higher costs in terms of fuel consumption, aircraft maintenance and pilot training. In order to overcome these disadvantages, a one-lever system for the control of the variable pitch turboprop aircraft propulsion system has been proposed. The one-lever approach, for given flight condition (required thrust, aircraft Velocity True Air Speed and ambient conditions) is able to determine blades pitch angle propeller rotational speed configuration in order to make sure that during the aircraft operations the propeller blades maintain the maximum airfoil efficiency. In this paper, the one lever control management system has been designed considering a model-based approach. Specifically, the turboprop propulsion system mathematical model represented the model reference of the one-lever model-based controller. In order to test the proposed one lever system, not only the turboprop propulsion system mathematical model had been developed, but the entire aircraft ecosystem has been taken into account. Such an aircraft whole system has been implemented in Simulink (C) framework including the aircraft dynamic model, the aircraft trajectory calculation and the ambient condition model. All the results have been remarkably encouraging, showing better performance in terms of efficiency and fuel consumption.

\section{References}

1. U. Ghezzi, Motori per Aeromobili, (Maggioli Editore, politecnica, 2008 - ISBN 8838741867)

2. R. Storm, M. Skor, L.D. koch, T. Benson, C. Galica, Pushing the Envelope: A NASA Guide to Engines (National Aeronautics and Space Administration, 2015)

3. A. Hasselrot, B. Montgomerie, FOI - Swedish Defence Research Agency pp. 1-66 (2005)

4. G. Zizzi, ed., Storia ed evoluzione della propulsione aerea (Stem Mucchi Editore, Modena, Italy, 1977 - ISBN B000Z9SPDU)

5. J.L. Musgrave, Single-Lever Power Control for General Aviation Aircraft Promises Improved Efficiency and Simplified Pilot Controls (National Aeronautics and Space Administration, 1997)

6. A. Lacivita, Master Thesis - Modeling and control of a turboprop with a variable pitch propeller (Politecnico di Bari, 2017) 
7. M.M. Procaccino, Master Thesis - Analisi delle prestazioni e strategia di controllo di un motore turboelica (Politecnico di Bari, 2016)

8. A. Andria, Master thesis - Simulazione Turboprop in ambiente Simulink (Politecnico di Bari, 2016)

9. J.F. Sellers, C. Daniele, Dyngen: A program for calculating steady-state and transient performance of turbojet and turbofan engines (National Aeronautics and Space Administration, 1975)

10. G. Rabagliati. - Scienze e Tecnologie Applicate. Trasporti e logistica (Simone Scuola, 2020 - ISBN 978-88-244-2857-6)

11. C.D.Leoni, Trasporto aereo ed aeroporti tra norme tecniche e regole di mercato. (CERTAM Editore, 2001)

12. Lozza, G. - Turbine a gas e cicli combinati (Progetto Leonardo, Bologna, 1996)

13. H. Saravanamuttoo, G. Rogers, H. Cohen e P. Straznicky - Gas turbine Theory (Addison Wesley Longman, 1996) 